\title{
WhatsApp and Wellbeing: A study on WhatsApp usage, communication quality and stress
}

\author{
Nicole Blabst \\ Ludwig-Maximilians-University Munich \\ nicole.blabst@campus.Imu.de
}

\author{
Sarah Diefenbach \\ Ludwig-Maximilians-University Munich \\ sarah.diefenbach@Imu.de
}

\begin{abstract}
A considerable part of everyday communication is online based nowadays. To imagine life without the daily (or even hourly) usage of WhatsApp seems impossible for many people. The present exploratory study $(\mathrm{N}=135)$ takes a closer look at the usage of WhatsApp and the psychological consequences. Our study highlights correlations and differences of the usage and experience of specific WhatsApp features (single chats and group chats, Last Seen and Read Receipts) with perceived communication quality and wellbeing, also drawing relations to psychological theory such as human needs framework and need to belong. A high number of single chats was positively correlated with perceived communication profundity but also with perceived stress, and waste of time. Moreover, wellbeing was affected by the individual usage mode and experience of WhatsApp features. For example, perceived stress was significantly higher among participants with active usage of Read Receipts than with passive usage and especially participants who feel stressed by Read Receipts, agreeing to be more relaxed without them, considered WhatsApp communication a waste of time. We discuss implications of our findings on the level of personal usage behaviour as well as $\mathrm{HCl}$ research and design in general. We highlight the challenges for the individual to customize technology to support a healthy use in daily life. Finally, the present study emphasizes the need for user experience evaluation on a fine-grained level, taking focus on single features and their consequences, and recognising how their activation or deactivation can eventually change the product character as a whole.
\end{abstract}

WhatsApp, Wellbeing, Usage mode, Communication quality, Last Seen, Read Receipts

\section{INTRODUCTION}

Next to the classic face-to-face communication or the good old phone call, instant messaging platforms like WhatsApp become a central communication channel and means to maintain relationships. Naturally, the inherent possibilities and features of instant messaging platforms also shape the way of communication and impose new rules of social interaction. For example, knowing that the other will see when you have read a message (Read Receipts), one might think twice before actually reading it. On a broader level, the question arises what this means for us and our interaction with others? How does the use of WhatsApp affect our wellbeing and how do specific WhatsApp features affect communication quality?

The present study explores WhatsApp and its features from the perspective of psychological wellbeing, based on empirical data from 135 WhatsApp users. A special focus lies on three options/features: Chats consisting only of two people, so called single chats and chats with more than two people, so called group chats. The other two features are technically supported help functions: Last Seen and Read Receipts (see Figure 1). Last Seen allows you to monitor the last time a communication partner opened WhatsApp. Read Receipts are being sent via the appearance of two blue tick marks.

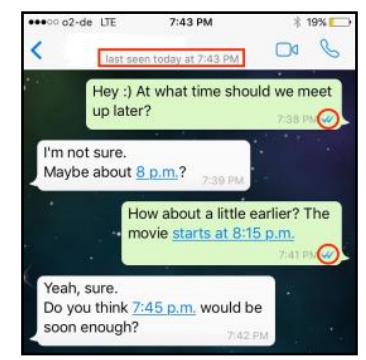

Figure 1: Screenshots of WhatsApp features Last Seen and Read Receipts 


\section{THEORETICAL BACKGROUND AND RESEARCH QUESTIONS}

The present study's interest on the effects of WhatsApp from the perspective of psychological wellbeing relates to prior research on technology use and wellbeing in the field of Human-ComputerInteraction $(\mathrm{HCl})$ as well as basic theory from psychology. In both fields, there is an emerging research interest in wellbeing, especially in the growing field of positive psychology. The definition of wellbeing is mostly tailored to context of research. In line with Burke and Kraut (2016) the present study takes a closer look at wellbeing components associated with perceived communication quality and wellbeing in the context of online communication. The components were assessed through perceived communication profundity, perceived stress, perceived meaningfulness and perceived waste of time.

In $\mathrm{HCl}$, prior research has been mainly focused on the influence of social networking services (SNSs) like Facebook on wellbeing (e.g. Burke \& Kraut, 2016; Sagioglou \& Greitmeyer, 2014). More time spent on Facebook is associated with a decline in mood mediated by the feeling of not having done anything meaningful (Sagioglou \& Greitmeyer, 2014). Moreover, the decline in wellbeing was explained by the use of Facebook as means of social comparison and not as platform for interaction (also see Karapanos, Teixeira \& Gouveia, 2016). It thus seems that compared to SNSs, instant messaging platforms like WhatsApp should create a more interactive kind of usage, whereby a focus on single chats or group chats, could play a crucial role. Especially single chats should be far more interactive whereas group chats might support a less interactive, Facebook like usage of just scrolling and possibly not even reading. In consequence, one focus of the present exploration are correlations between the number of single/group chats and perceived communication quality and wellbeing.

Pielot, de Oliveira, Kwak and Oliver (2014) revealed that the usage of Last Seen can create social pressure. Having cues about the last time someone was online, generates expectations for oneself to give answers more promptly. Additionally, the sender expects to receive an answer more quickly if it is possible to check the last time someone was online. Those findings can be transferred to the impact of Read Receipts, as this feature is similar to Last Seen. In line with that, Ahad and Lim (2014) discovered that Last Seen and Read Receipts can promote addictive-like checking behaviour. Church and Oliveria (2013) additionally remarked that Read Receipts need to be treated more carefully, as they only state that the message was opened and not necessarily read. Consequently, this could foster inappropriate expectations from the sender of the message. Thus, another research interest of the present study relates to correlations between the usage and experience of Last Seen and Read Receipts and perceived communication quality and wellbeing.

Finally, the present study has relations to the human needs framework (Sheldon, Elliot, Kim, \& Kasser, 2001) and need to belong (Baumeister \& Leary, 1995). Concerning the human needs framework especially the need for relatedness is in focus. Prior research revealed that the need for relatedness is a predictor for WhatsApp use, while Facebook usage can be predicted by the need for self-esteem (Karapanos, Teixeira \& Gouveia, 2016). Another important aspect is the need to belong (Baumeister \& Leary, 1995). Especially profound communication with strong ties should foster the need to belong. Research on Facebook already revealed similar findings, thus WhatsApp should be even more likely to provide a basis for strong tie communication through the usage of single chats (Burke \& Kraut, 2016; O'Hara, Massimi, Harper, Rubens, \& Morris, 2014). To explore this aspect, the present study focused on several components of communication quality and indicators of felt relatedness within a conversation, such as perceived communication profundity and meaningfulness.

\section{METHOD}

\subsection{Participants and Procedure}

The study was carried out online and 135 people (114 males, 20 females, 1 other) completed the survey. The age range was between 16 and 65 years $(M=26.6, S D=8.1)$. Participants were gathered on a convenient basis through Facebook groups associated with psychological studies and through university boards. The questionnaire took about 10 to 15 minutes to complete. Participants were pre-screened for active usage of WhatsApp and their smartphone had to be within their reach.

\subsection{Questionnaire}

The questionnaire broadly focused on two main aspects: First, usage and experience of WhatsApp features, whereby especially the usage and experience of the WhatsApp features "single chats and group chats" as well as "Last Seen and Read Receipts" were further illuminated. Second, perceived communication quality and wellbeing, posing questions on perceived communication profundity, perceived stress, perceived meaningfulness and perceived waste of time. The concrete measures for both aspects are described in the next sections. Besides, participants were asked to provide demographic core data. However, due to the limited capacity of the present report, only a selection will be discussed. 
3.2.1. Usage and experience of WhatsApp features To measure the usage and experience of WhatsApp features, participants were asked to provide information on:

- Number of single chats and group chats. They were asked to open WhatsApp to count their single and group chats of the last three days including the present day.

- Usage of Last Seen and Read Receipts. Participants had to answer questions concerning their usage (choosing between the options turned off, passive usage, active usage). The goal was to not only take a closer look at the technical usage - simply having them turned off/on - but asked participants to further describe how they used them. The users had to state if they had turned them on, but were not looking when someone was last online or if someone had already read their message (passive usage) or if they were actively using those features (active usage).

- $\quad$ Experience of Last Seen and Read Receipts. They were asked whether it "would be more relaxed without Last Seen/Read Receipts" (seven-point Likert scale, strongly agree to strongly disagree).

\subsubsection{Perceived communication quality and wellbeing}

To assess the perceived communication quality and wellbeing participants provided the following ratings:

- A rating on perceived communication profundity of WhatsApp chats, assessed by a slide bar (100 units, superficially vs. profoundly).

- A single item on perceived meaningfulness of the time spent on WhatsApp (seven-point Likert scale, strongly agree to strongly disagree).

- A single item on perceived stress (seven-point Likert scale, strongly agree to strongly disagree).

- A single item on perceived waste of time through the usage of WhatsApp (seven-point Likert scale, strongly agree to strongly disagree).

\section{RESULTS}

\subsection{Descriptive Analyses}

The mean number of single chats was $8.07(S D=$ 4.86 ), the mean number of group chats was 2.42 $(S D=1.96)$. Table 1 depicts the number of WhatsApp chats divided by sex and age groups. On average, women reported a higher number of single chats than men, whereas men reported a higher number of group chats than women. Also, participants younger than 25 years reported a larger number of chats than older participants. However, none of these differences were on a statistically significant level.

Concerning the frequencies of usage of different WhatsApp features, roughly $80 \%$ were using Read Receipts and about $60 \%$ Last Seen, and the majority agreed to knowing how to turn those features off (roughly $80 \%$ ). $42 \%$ tended to agree to the item "it would be more relaxed without Read Receipts" and gave a rating in the upper scale range above $4(M=$ 3.96 ); $46 \%$ tended to agree to "it would be more relaxed without Last Seen" $(M=3.83)$, and both items were highly correlated $(r=.717, \mathrm{p}<.001)$.

Table 2 depicts the average ratings on perceived communication quality and wellbeing. Perceived communication profundity and meaningfulness are on a median level. Compared to this, the ratings on perceived waste of time and perceived stress show a more definite tendency. While most users would not consider WhatsApp chats as a waste of time, most users consider them as quite stressful at the same time.

Table 1: Usage intensity of WhatsApp: Number of chats by sex and age groups

\begin{tabular}{|c|c|c|c|c|}
\hline $\begin{array}{l}\text { Chat } \\
\text { type }\end{array}$ & Sex/Age & Median & Mean & SD \\
\hline \multirow{5}{*}{$\begin{array}{l}\text { Single } \\
\text { Chats }\end{array}$} & Female & 7.00 & 8.24 & 4.96 \\
\hline & Male & 5.50 & 7.25 & 4.36 \\
\hline & Other & 5.00 & 5.00 & . \\
\hline & Age group 18-24 & 8.00 & 8.77 & 4.87 \\
\hline & Age group 25-65 & 6.00 & 7.24 & 4.77 \\
\hline \multirow{5}{*}{$\begin{array}{l}\text { Group } \\
\text { Chats }\end{array}$} & Female & 2.00 & 2.32 & 1.84 \\
\hline & Male & 2.50 & 3.10 & 2.53 \\
\hline & Other & 1.00 & 1.00 & . \\
\hline & Age group 18-24 & 3.00 & 2.85 & 2.05 \\
\hline & Age group 25-65 & 1.50 & 1.92 & 1.73 \\
\hline
\end{tabular}

Table 2: Perceived communication quality and wellbeing

\begin{tabular}{|l|l|l|l|}
\hline \multicolumn{1}{|c|}{ Rating/Item } & Median & Mean & SD \\
\hline $\begin{array}{l}\text { Perceived communication } \\
\text { profundity (scale 1-101) }\end{array}$ & 46.50 & 46.72 & 21.55 \\
\hline $\begin{array}{l}\text { Perceived meaningfulness } \\
\text { (scale 1-7) }\end{array}$ & 4.00 & 4.33 & 1.40 \\
\hline $\begin{array}{l}\text { Perceived waste of time } \\
\text { (scale 1-7) }\end{array}$ & 3.00 & 3.29 & 1.69 \\
\hline $\begin{array}{l}\text { Perceived stress } \\
\text { (scale 1-7) }\end{array}$ & 5.00 & 4.33 & 1.81 \\
\hline
\end{tabular}

\subsection{Correlational Analyses}

A correlational analysis of the relationships between usage and experience of WhatsApp features, and perceived communication quality and wellbeing revealed several significant correlations. The 
number of single chats was positively correlated with perceived stress $(r=.20, p<.05)$, perceived communication profundity $(r=.34, p<.01)$, and perceived waste of time $(r=.18, p<.05)$. The number of group chats also positively correlated with perceived waste of time $(r=.18, p<.05)$. Data revealed no significant correlations between number of chats and meaningfulness of communication.

Also, the individual experience of WhatsApp features was correlated with perceived communication quality. There was a negative correlation between agreeing to the item "it would be more relaxed without Last Seen" and meaningfulness $(r=-.31, p<.01)$. Agreeing to the item "it would be more relaxed without Read Receipts" positively correlated with perceived waste of time $(r=.20, p<.05)$. Data revealed no significant correlations between the individual experience of WhatsApp features and perceived stress or perceived communication profundity.

\subsection{Analyses of variance}

The found correlations between number of single chats and perceived stress, perceived communication profundity and perceived waste of time was further explored by one way ANOVAs, with the categorized number of single chats as independent measure. A significant effect of chat number emerged for perceived communication profundity, $F(4,130)=5.98, p<.001$, and a marginally significant effect for perceived stress, $F(4,130)=2.13, p=.081$. As shown by the descriptive data in Figure 2, there is no infinite increase of perceived communication profundity. Instead, it reaches a maximum among participants with 9-10 single chats, and then drops for participants with more than ten chats. Interestingly, there is a similar pattern for perceived stress, i.e., a drop in stress values after reaching a maximum for people with 9-10 chats (see Figure 3 ).

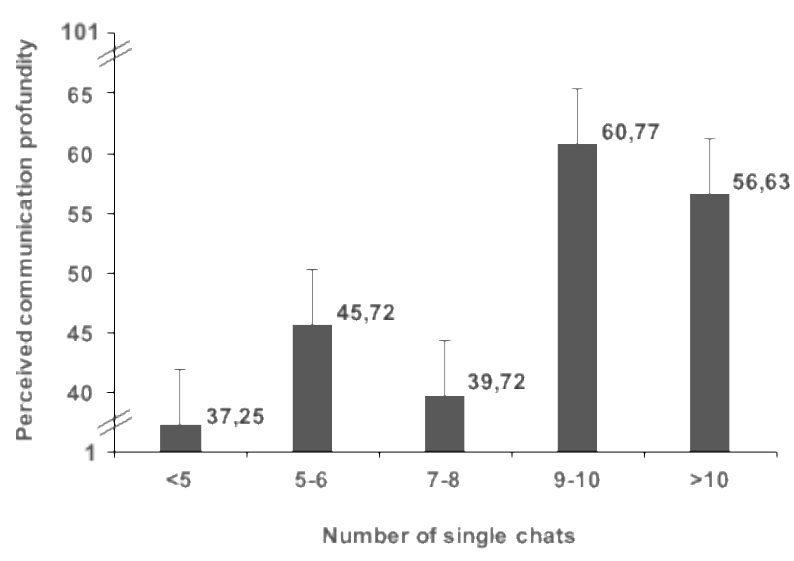

Figure 2: Means and standard errors of perceived communication profundity, depending on the number of single chats.

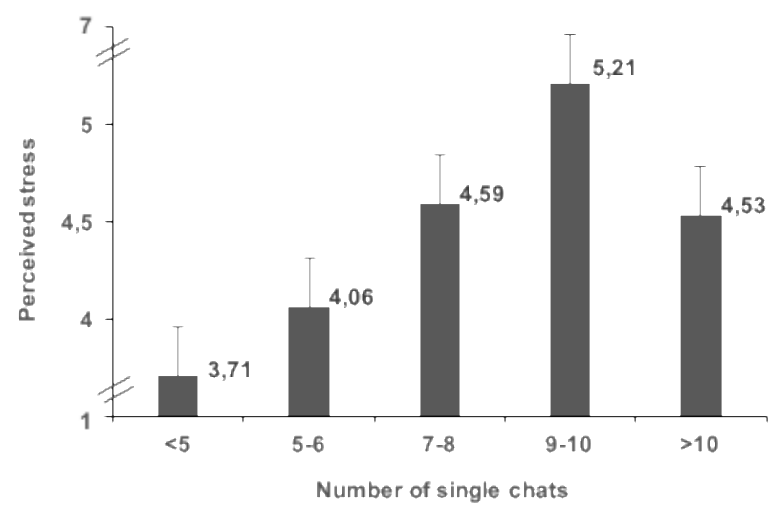

Figure 3: Means and standard errors of perceived stress, depending on the number of single chats.

No significant differences in the determinants of perceived communication quality and wellbeing in relation to the different usage modes of Last Seen (Turned off, passive usage, active usage) could be observed. However, a one-way ANOVA revealed significant differences in perceived stress depending on the usage of Read Receipts (Turned off, passive usage, active usage), $\mathrm{F}(2,134)=3.514, p=.033$, $\eta p^{2}=.051$. Figure 4 shows mean values and standard errors of perceived stress for the different usage modes. Post-hoc analyses using Tukey's HSD indicated that perceived stress of participants with active usage was significantly higher than perceived stress of participants with passive usage $(p=.025)$, but perceived stress did not differ significantly between participants with turned off Read Receipts and passive usage $(p=.301)$ or between participants with turned off Read Receipts and with active usage $(p=.832)$.

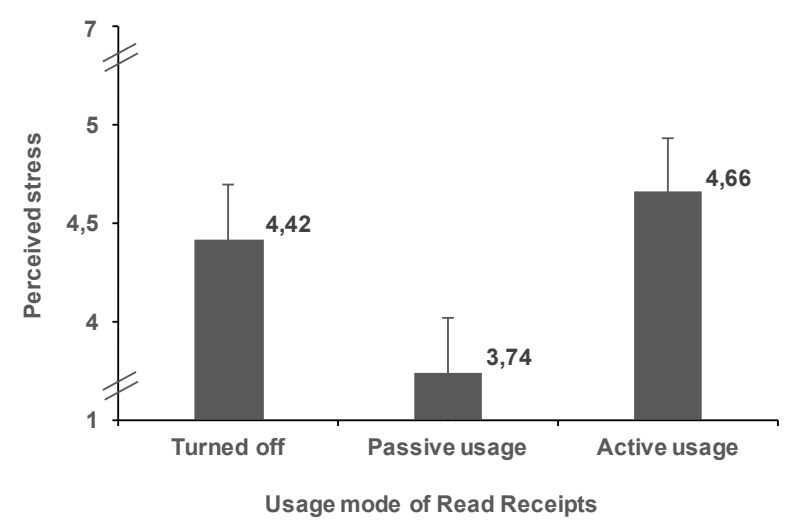

Figure 4: Means and standard errors of perceived stress, depending on the usage mode of Read Receipts.

\section{DISCUSSION}

Overall, the perceived meaningfulness and perceived communication profundity of WhatsApp 
chats was on a medium level. Furthermore, user ratings suggested that WhatsApp chats were generally appreciated, i.e., most users did not consider them as a waste of time. However, the majority of participants also reported stress values in the higher scale ranges related to WhatsApp use. This conjunction might express an ambivalent experience often related to using communication technology in general. One would not want to miss them, but one also feels an increasing pressure to handle the many opportunities and demands posed by different channels of communication. In consequence, it often seems a challenge to find the right balance between digitally mediated meaningful and stressful moments.

Correlational data revealed that this particularly pertains to users communicating within a lot of single chats. Though a higher number of single chats was correlated with higher perceived communication profundity, it was also related to higher feelings of stress and perceived waste of time. While a certain level of chat intensity may express the relevance of WhatsApp as a true medium of conversation, mediating profound communication, there could be a "saturation point" where no more chats can be meaningfully integrated, as also indicated by the drop in perceived communication profundity for participants handling more than ten single chats. However, further research is necessary to explore such relationships in detail and create a broader empirical basis for generalizations. In the following, we discuss the present findings with regards to previous empirical findings and theoretical concepts.

\subsection{Number of chats and perceived communication quality and wellbeing}

Correlational data revealed that using WhatsApp to communicate within a lot of single chats is related to feeling stressed. However, the perceived communication profundity was also rated to be higher. Still, communication within a lot of single and group chats is considered to be a perceived waste of time. Thus, WhatsApp has the inherent potential for profound communication in single chats and can foster the need for relatedness as a predictor of WhatsApp usage in line with research of Karapanos, Teixeira and Gouveia (2016). Further, the need to belong (Baumeister \& Leary, 1995) also seems to be satisfied through the profound communication as it provides a basis to form secure and committed bonds as already stated by O'Hara, Massimi, Harper, Rubens and Morris (2014). Nevertheless, the results of the present study also substantiate the findings of research on Facebook (e.g. Burke \& Kraut, 2016; Sagioglou \& Greitmeyer, 2014). A larger number of chats can make people feel stressed and the time spent is rather seen as a perceived waste of time. This mirrors the negative effects of an increased Facebook usage on mood and the mediating feeling of not having done anything meaningful, found by Sagioglou and Greitmeyer (2014). Burke and Kraut (2016) revealed that in the general context of Facebook usage, targeted, composed communication with strong ties is especially beneficial to wellbeing. Even though WhatsApp is only an instant messaging platform, this might nevertheless also be true for the profound communication in single chats.

\subsection{Individual usage and experience of Last Seen and Read Receipts and perceived communication quality and wellbeing}

As shown by the analyses of different usage modes of Read Receipts, perceived stress of participants with active usage was significantly higher than perceived stress of participants with passive usage. Those findings are in line with previous research (Ahad \& Lim, 2014; Church \& Oliveria, 2013; Pielot, de Oliveira, Kwak \& Oliver, 2014), reporting that actively looking if a sent message has already been read, can create an atmosphere of stress.

In the present study, especially participants who reported to feel stressed through WhatsApp features such as Last Seen and Read Receipts, also experienced WhatsApp usage as not having done anything meaningful and a waste of time. A critical attitude against status notifications and read receipts was already found in line with previous research (Ahad \& Lim, 2014; Church \& Oliveria, 2013; Pielot, de Oliveira, Kwak \& Oliver, 2014). Though surely having practical advantages in particular situations, people also experience the critical effects and negative emotions related to such features, leading to the result that some people would rather not like to see if someone has already read their message or see the last time that someone was online on WhatsApp. In sum, especially people, who are sensitive for the social pressure of receiving answers or having to give them quickly, would probably profit from not using Last Seen and Read Receipts at all.

\subsection{Overload and social networking service (SNS) fatigue}

Overall, the present findings suggest that communication technologies such as WhatsApp provide a channel for meaningful and emotional exchange between people but that a high number of chats and a high sensitivity to status/read receipt features can also be associated with negative psychological consequences for the user. Those findings are in line with Lee, Son and Kim (2016) who outlined that information, communication and system feature overload can result in SNS fatigue. This can actually lead to the consequences of taking a break or suspending activities on SNS. Even though WhatsApp is primarily a platform for communication, it can quickly create overload for users and thus requires a deliberate use. 


\section{LIMITATIONS AND FUTURE WORK}

The results provide a first assessment of different WhatsApp features and their dangers respectively boost for perceived communication quality and wellbeing. However, there are limitations to the exploratory study. Due to the cross-sectional approach no causal assumptions can be made. Further studies might explore the correlations in a longitude design to shed light on causal relations. To observe a broader variety of significant correlations a bigger sample size might be needed. Additionally, the study was conducted online, thus, some participants might not have been fully committed to answer all of the questions considerately.

\section{CONCLUSION}

WhatsApp is a major part of online communication nowadays with a considerable growth rate of users. Even though profound communication is possible, one might consider that less can actually be more. Feeling stressed by features like Read Receipts should not remain a permanent condition. There are solutions like turning them off or using them more deliberately. WhatsApp offers several feature that need to be customized by the individual to support a healthy use of technology in daily life.

On a more general level, from the perspective of $\mathrm{HCl}$ research and UX evaluation, the present example of our study on WhatsApp demonstrates how the usage and experience of technology can be a rather individualistic endeavour, only revealed by finegrained user experience evaluation. Depending on how the individual actually uses the product (e.g., active versus passive usage of Read Receipts), the resulting user experience may be fundamentally different, and dominated by positive or negative emotions. Thus, the present study highlights the need for UX evaluation to not only consider a product a whole, but also the appropriations through the user and their consequences for the users' wellbeing. In conclusion, a topic of increasing importance in $\mathrm{HCl}$ research is not only to understand what users experience when interacting with technology, but also to use such insights in the sense of transformative consumer research improving consumers' wellbeing (Mick, Pettigrew, Pechmann, \& Ozanne, 2012), which also means to assist users how to integrate such technology into their life in a way that is good for them.

\section{REFERENCES}

Ahad, A. D., \& Lim, S. M. A. (2014) Convenience or nuisance?: The 'WhatsApp' dilemma. ProcediaSocial and Behavioral Sciences, 155, 189-196. http://dx.doi.org/10.1016/j.sbspro.2014.10.278
Baumeister, R. F., \& Leary, M. R. (1995) The need to belong: Desire for interpersonal attachments as a fundamental human motivation. Psychological Bulletin, 117(3), 497.

Burke, M., \& Kraut, R. E. (2016) The Relationship between Facebook Use and Well-Being depends on Communication Type and Tie Strength. Journal of Computer-Mediated Communication, 21(4),265-281.

http://dx.doi.org/10.1111/jcc4.12162

Church, K., \& de Oliveira, R. (2013) What's up with whatsapp?: comparing mobile instant messaging behaviors with traditional SMS. 15th international conference on Human-computer interaction with mobile devices and services, Munich, August 30th, 2013, (pp. 352-361). ACM, Munich.

Karapanos, E., Teixeira, P., \& Gouveia, R. (2016) Need fulfillment and experiences on social media: A case on Facebook and WhatsApp. Computers in Human Behavior, 55, 888-897. http://dx.doi.org/10.1016/j.chb.2015.10.015

Lee, A. R., Son, S. M., \& Kim, K. K. (2016) Information and communication technology overload and social networking service fatigue: A stress perspective. Computers in Human Behavior, 55, 51-61. http://dx.doi.org/10.1016/j.chb.2015.08.011

Mick, D. G., Pettigrew, S., Pechmann, C. C., \& Ozanne, J. L. (Eds.). (2012). Transformative consumer research for personal and collective well-being. New York, NY: Routledge.

O'Hara, K. P., Massimi, M., Harper, R., Rubens, S., \& Morris, J. (2014) Everyday dwelling with WhatsApp. In Proceedings of the 17th ACM conference on Computer supported cooperative work \& social computing, Baltimore, Maryland, 14-19th February 2014, (pp. 1131-1143). ACM, Baltimore, http://dx.doi.org/10.1145/2531602.2531679

Pielot, M., de Oliveira, R., Kwak, H., \& Oliver, N. (2014) Didn't You See My Message? Predicting Attentiveness to Mobile Instant Messages. Poster presented at $\mathrm{CHI}$ 2014, Toronto, Canada. http://dx.doi.org/10.1145/2556288.2556973

Sagioglou, C., \& Greitemeyer, T. (2014) Facebook's emotional consequences: Why Facebook causes a decrease in mood and why people still use it. Computers in Human Behavior, 35, 359-363. http://dx.doi.org/10.1016/j.chb.2014.03.003

Sheldon, K. M., Elliot, A. J., Kim, Y., \& Kasser, T. (2001) What is satisfying about satisfying events? Testing 10 candidate psychological needs. Journal of personality and social psychology, 80(2), 325. 